\title{
STENOSIS OF THE SUPERIOR MESENTERIC ARTERY
}

\author{
By G. E. Mavor, M.A., M.D., Ch.M., F.R.C.S.E. \\ Aberdeen Royal Infirmary
}

It has been known for many years that disease of the superior mesenteric artery can produce abdominal pain and other chronic symptoms in the absence of gangrene of the bowel or peritonitis (Klein, I92I ; Conner, 1933; Hertzler, I935; Seymour and Liebow, 1937), and that inevitably these symptoms terminate in acute bowel infarction. However, it is only recently, as the feasibility of direct arterial surgery has become apparent, that these clinical facts have acquired new significance and more attention has been paid to the pathological background of midgut ischaemia and the nature and extent of the occlusion in the superior mesenteric artery.

The chronic symptoms of midgut ischaemia have been variously referred to as 'intermittent mesenteric claudication' (Klein, 192I), ' abdominal angina' (Larson, I93I), 'abdominal claudication ' (Conner, 1933), ' mesenteric stroke' (Klass, 1953), and 'intestinal angina' (Lancet, 1958). None of these terms seems as precise or as accurate as that of ' chronic midgut ischaemia.'

\section{General Considerations}

Over the past Io years the incidence of mesenteric infarction has been steadily rising, and according to the Registrar-General's report has now reached over 400 cases each year. This serious condition carries a very high mortality, reaching 80 to 90 per cent. (Moore, 194I ; Forty, 1957; Berman and Russo, 1950; McClenahan and Fisher, 1948). The advent of the anticoagulants and the antibiotics has not materially altered the position. In the presence of bowel gangrene, resection has been the traditional treatment and little attention has been paid to the superior mesenteric artery. In fact the possibilities of direct arterial surgery in the presence of severe bowel ischaemia have not as yet been fully explored.

A major cause of the high mortality in mesenteric infarction lies in the fact that for various reasons surgical treatment has awaited the development of the acute symptoms indicating infarction of the bowel. Obviously this is unavoidable if the symptoms are of acute onset without any antecedent history. But there is no certainty as to how often this is indeed the case. In many recent reviews of acute mesenteric infarction, no mention is made of prodromal attacks, although as long ago as 1935 Hertzler stated that 'incomplete occlusion of the superior mesenteric artery is the basis of the prodromal phases in mesenteric vascular occlusion. It is obvious that the beginnings date back several weeks or months and that acute symptoms date from the time the affected vessel finally obstructs the circulation.' It would seem that careful history taking will often reveal premonitory symptoms and several such cases have been recently described (Benjamin, 1948; Mandel 1957; Mikkelsen, I957; Mavor and Michie, 1958). Inevitably such symptoms vary in degree, and in certain instances may be so slight that they are overlooked by the patient. The incidence of their occurrence is thus difficult to assess and as yet there is insufficient information on this point in the literature. In a careful paper, Dunphy and Whitfield (1940) found that seven patients out of I2 with fatal mesenteric occlusion had prodromal attacks, and in a recent personal series of 10 cases, six patients were noted to have previous symptoms indicative of chronic midgut ischaemia. The incidence may thus be high enough to improve the outlook in mesenteric infarction by enabling satisfactory palliative surgical treatment to be undertaken at a stage prior to complete occlusion of the vessel and infarction of the bowel, always provided a proper appreciation of the significance of the prodromal symptoms exists.

\section{Pathology}

In the presence of gross narrowing or segmental occlusion of a large artery the degree of peripheral ischaemia depends on many factors. These include the site, extent and rapidity of the narrowing or occlusion, the age and physical condition of the patient, relating in most instances to his cardiac state, the extent of the disease in the peripheral 
arterial tree, and the collateral circulation. As regards the latter, the anatomical arrangements in the superior mesenteric arterial system are such that with stenosis or occlusion of the main vessel, the intestinal blood supply must always be regarded as critical. The main collateral vessels are limited to the inferior pancreatico-duodenal artery, which anastomoses with the superior pancreatico-duodenal branch of the gastro-duodenal artery, and the middle colic artery, which anastomoses with the left colic branch of the inferior mesenteric artery. The usual sequel to occlusion of the superior mesenteric artery is infarction of the bowel, although instances of occlusion with survival have been reported (Klein, I92I; Moore, I94I). In such cases obliteration has probably been gradual; however, even acute occlusion, such as results from embolism, may not prove fatal (Klein, I92 I). The existence of subperitoneal and retroperitoneal plexuses (Turner, 1863; Burt et al., 1952) can greatly increase the communications between somatic and visceral vessels, permitting an adequate supply to the stomach, small bowel and proximal colon, when both coeliac and superior mesenteric arteries are occluded at their origins (Chiene, r869). Rob and Owen (1956) recently reported a case in which at operation all three branches of the coeliac axis and the superior mesenteric artery were thrombosed at their origins and could be divided with impunity. In such circumstances the subcostal and lumbar arteries may play a r'le in the collateral blood supply.

It would appear, then, that the nature of the interference with the intestinal blood supply which will give rise to symptoms of chronic midgut ischaemia varies in individual cases. In many instances gross stenosis of the superior mesenteric artery apparently diminishes blood flow sufficiently to produce symptoms (Johnson and Baggentoss, 1949; Mavor and Michie, 1958). On other occasions, stenosis of the artery has to be associated with occlusion of the coeliac axis before blood flow is reduced to critical levels (Seymour and Liebow, 1937; Mandel, 1957; Mikkelsen, I957; Cooke, 1958), and even more rarely complete occlusion may occur with only chronic symptoms resulting.

There seems little doubt that the common lesion is stenosis of the superior mesenteric artery secondary to atheroma: the coeliac axis may or may not be involved. Klein (192I) realized this, but the significance of his contribution is only now being recognized. The anatomy of the superior mesenteric artery is interesting in that the vessel generally has a large main trunk, the branches of which are few in number. They include, however, the important middle colic artery. Below the lower border of the pancreas, the vessel

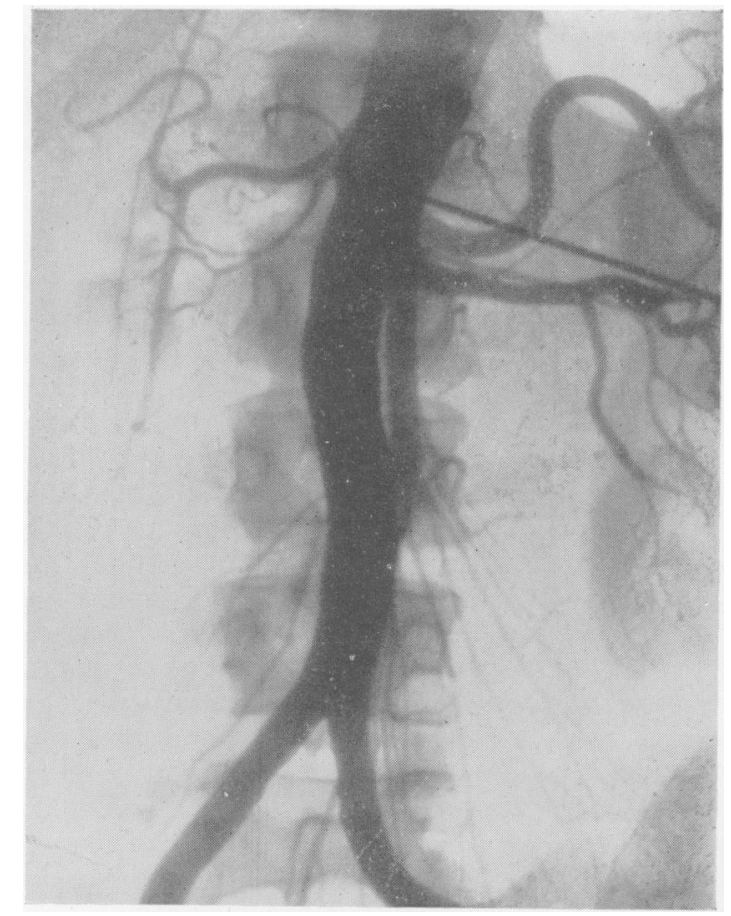

FIG. 1.-Translumbar aortogram showing the large main trunk of the superior mesenteric artery and the manner in which it may rapidly divide into jejunal and ileal branches.

divides into numerous jejunal and ileac branches and becomes itself greatly reduced in size (Fig. I). The observations of Carucci (1953) indicate that here, as elsewhere in the body, atheroma may for a time be largely restricted to the main vessel, while the peripheral arteries escape the disease. He described a study of 50 post-mortem cases, 90 per cent. of which were over 50 years of age, noting that there was no gross evidence of atheroma or sclerosis in any case in the mesenteric vessels beyond the main trunk, whereas 22 out of the 50 cases showed evidence of atheroma at or near the origin of the vessel.

The precise pathological picture in 60 cases of superior mesenteric artery occlusion was detailed by Johnson and Baggentoss in 1949. Of the 60 cases, they noted that in 22 the lesion was at the origin or in the main stem of the vessel. In 18 others, the main trunk was also possibly involved. In 17 , however, they reported involvement of the small branches in the mesentery. More information is required on this matter, as it may have a significant bearing on possible surgical treatment.

In some cases narrowing of the superior mesenteric artery is not related to atheroma of the vessel itself, but to atheroma of the aorta with or without associated mural thrombosis. Johnson and 
Baggentoss (I949) mention this possibility, noting it in three instances, and more recently Mavor and Michie (1958) have described a case. Such an occurrence appears rare, as neither Leriche and Morel (1948), nor Burt, Learmonth and Richards (1952) in reviewing their cases of aortic thrombosis mention midgut ischaemic symptoms.

There is no evidence as yet that symptoms of chronic midgut ischaemia can occur on the basis of pathology other than atheroma. Polyarteritis nodosa affects the mesenteric vessels in 30 per cent. of all cases (Arkin, 1930), but in only about ro per cent. of these is occlusion of the main trunk of the superior mesenteric artery found (Johnson and Baggentoss, 1949). Even so the nature of the arteritis is such that occlusion is likely to be complete and extensive from the outset. Dunphy and Whitfield (1940) and later Berman and Russo (1950) suggested that spasm of the smaller mesenteric vessels played a role in the symptomatology, but there seems no good reason for suggesting this, nor is there supportive evidence available. It is also difficult to visualize how small, non-fatal peripheral occlusions, due either to embolism or thrombosis, can explain the constant day-to-day symptoms characteristic of the syndrome. The clinical evidence suggests that the symptoms are due to inadequate intestinal blood flow, although the exact mechanism involved is not established.

\section{The Clinical Picture}

The clinical picture in chronic midgut ischaemia is so characteristic that in many cases the diagnosis can be reliably made on the basis of the symptomatology. The following is an illustrative case report.

\section{Case Report}

A man, aged forty-nine, was first seen because of lower abdominal pain. Four months previously he had a sudden onset of severe lower abdominal pain. This was associated with vomiting, and the next day he passed three or four dark fluid stools. The severity of the pain subsided after several days, but some pain remained present daily. This pain, situated in the lower abdomen, was continuous in nature and aggravated by a heavy meal, particularly of meats or fats. He had no abdominal colic, no night pain, and no back pain. Occasionally after a large meal he vomited. During this period he tended to be constipated, but since the original episode had had two episodes of diarrhoea, each lasting a few days. His appetite was poor and he lost $2 \mathrm{I} \mathrm{lb.} \mathrm{(9.5} \mathrm{kg.)} \mathrm{in} \mathrm{weight} \mathrm{in} \mathrm{four} \mathrm{months.}$

On examination he was a thin man of good colour. There were no findings of significance in the abdomen. Rectal examination was negative. Stool benzidine examination was positive. Haemoglobin was 92 per cent., and a barium-meal and follow-through examination showed no abnormality.

He was seen twice during the next three months. Diarrhoea had become more persistent and he passed two or three stools daily. These were watery but well coloured. The abdominal pain $\stackrel{5}{?}$ persisted, being present every day. It came on $\bar{c}$ regularly within a few minutes of eating, and subsided after a few hours. The relationship between pain and eating was clearly perceived by the patient. There was no further vomiting. of Weight loss continued, the total loss over seven months being $35 \mathrm{lb}$. (15.9 kg.). On one occasion rectal examination revealed soft yellowish faeces which suggested fat malabsorption. Fatty acids were present on examination. Stool benzidine examination continued to be positive, and a further haemoglobin estimation was 90 per cent. Barium enema showed no abnormality.

Seven months after the onset of symptoms and while awaiting admission to hospital for investigation, he entered as an emergency case, complaining of severe abdominal pain. For the previous six days he had constant lower abdominal pain of great severity. He vomited brownish material several times. He had had no adequate bowel movement over the previous five days, although he had passed some dark fluid faeces from time t\& time. The abdominal pain and vomiting hae increased over the previous 24 hours, and abdominal distension had become apparent.

On examination he was emaciated and suffering severe pain. The blood pressure was $160 / 100 \mathrm{~mm}$. $\mathrm{Hg}$., and the pulse rate 90 a minute. His extremities were cold, there was peripheral cyanosis, and venous filling was poor. Other significant findings were restricted to the abdomen, which was distended. There was slight lower abdominal tenderness, but no rigidity and no palpable mass. The abdomen was tympanitic on percussion and quiet, with occasional high-pitched bowel sounds on auscultation. The bladder was not distended and the rectum was empty.

The patient was given 10,000 units of heparin and intravenous therapy was begun. A Ryle tube was passed and $120 \mathrm{ml}$. of dark brown fluid aspirated from the stomach. At laparotomy the small bowel (excluding the duodenum), caecum, and ascending colon were gangrenous. Peritonitis was well established. There was no pulsation in the superior mesenteric artery, which appeared thrombosed from its origin. No definitive surgical procedure was undertaken. The patient died eight hours later.

\section{Post-Mortem Findings}

The only abnormal findings were related to the abdomen. The peritoneal cavity contained a 


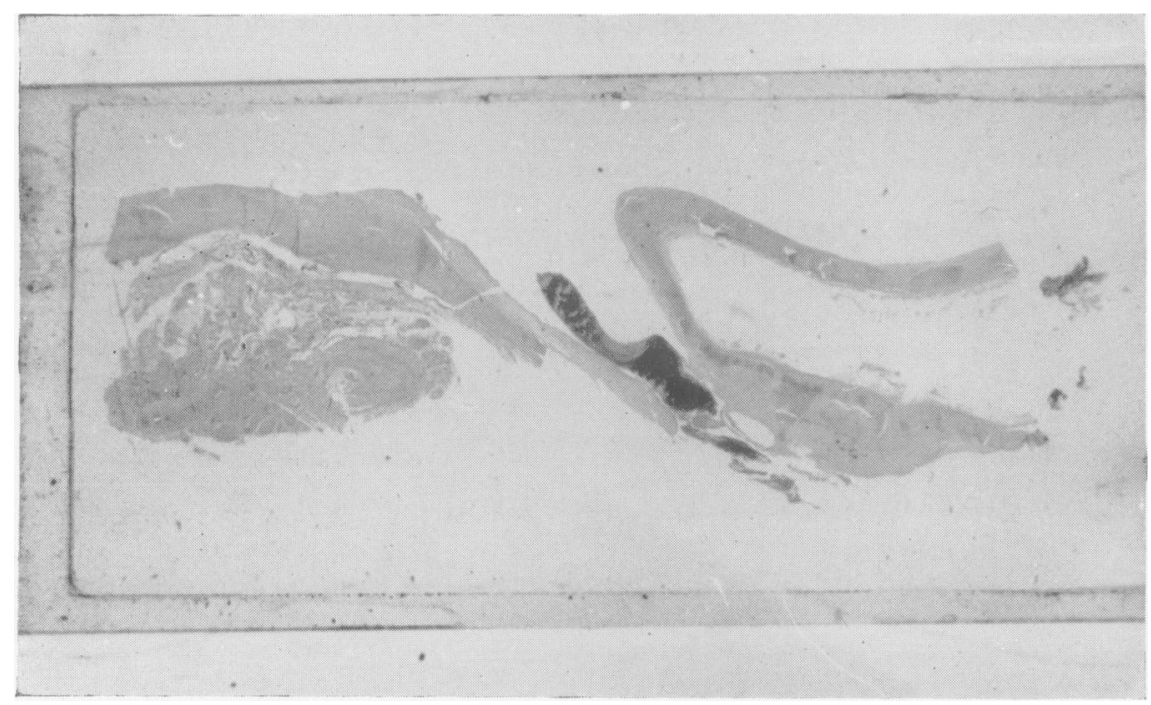

Fig. 2.- Section (actual size) through the superior mesenteric artery showing lumen just distal to origin narrowed by atheroma and occluded by fresh thrombus.

small amount of blood-stained serous fluid, and the entire small bowel and proximal half of the large bowel were necrotic. This extensive infarction of the bowel was due to thrombosis occluding the orifice of the superior mesenteric artery. In addition to the recent thrombus at the orifice, there was just inside the orifice an older organized layer of thrombus which was partly occluding the vessel wall (Fig. 2). Beyond this, the artery was free of atheroma and contained only post-mortem clot. The abdominal aorta and the coeliac axis were healthy.

\section{Symptomatology}

The most typical feature of the symptomatology is the pain. Initially the pain may be fairly mild, but it is generally present throughout the day. The overall picture is one of a steady unrelenting increase in severity as the weeks or months pass. It is deep-seated in the epigastrium or mid-abdomen, well-localized and with constant relationship to meals. It comes on soon after eating, sometimes within minutes, and lasts several hours. On other occasions there may be a lapse of an hour or two before its onset. The amount of food taken is also important, the pain generally being most severe following a large meal. The association between the onset of pain and the ingestion of food is rapidly perceived by the patient, who consequently rapidly loses his appetite and may become literally afraid to eat. Under these circumstances weight loss, always a feature of the illness, may become considerable. In several reported cases the pain is so constant in its daily occurrence that the patient is considered neurotic (Sedlacek and Bean,
I957), a conclusion seemingly supported by the striking contrast between the severity of the pain and the absence of physical signs. The pain is apparently true visceral pain and objective abdominal findings such as tenderness and muscular spasm are not found. The mechanism of its production remains unexplained, although it is obviously associated with ischaemia which becomes relatively more marked after meals.

Vomiting is uncommon, but it may occur when the period of chronic ischaemia is punctuated by acute ischaemic episodes leading to symptoms such as bloody diarrhoea and peripheral vascular collapse (Warburg, 1905; Sedlacek and Bean, 1957). When these acute symptoms occur at the outset of the illness, as in the above case, the subsequent development of chronic ischaemia is more easily recognized.

An altered bowel habit is a common finding in the disease. Klein (192I) mentioned that in the early stages the patient is often constipated, but this is by no means usual. Latterly, diarrhoea is common and may become very troublesome (Mavor and Michie, 1958). This persistent diarrhoea is not the result of acute ischaemia, but, as suggested by the character of the stools, is due to fat malabsorption. Klass (1953) has mentioned that absorption defects of the small bowel may occur in patients who have survived superior mesenteric arteriotomy, and Shaw and Maynard (1958) have reported a successful case of superior mesenteric endarterectomy in which the acute ischaemia caused absorption defects for fat, protein and carbohydrate. Together with the anorexia, the defective absorption contributes to the weight 
loss, which in the later stages may be so great as to cause death from inanition (Berman and Russo, 1950). The experimental work of Klein (192 I) has shown that in the presence of ischaemia the intestinal mucosa and submucosa suffer most severely. Mucosal ulcerations and widespread submucosal fibrosis are common findings. This readily explains the malabsorption and also accounts for the presence of occult blood in the stools in clinical cases.

\section{Investigation and Diagnosis}

Just as clinical examination is unrewarding, so the usual routine biliary and gastro-intestinal $\mathrm{X}$-ray studies generally show no abnormality, although occasionally the small bowel pattern on barium meal may suggest some dysfunction (Mandel, 1957). Specific investigation, such as superior mesenteric arteriography, has its limitations, largely related to the overlay of the main trunk of the vessel on the aorta (Fig. I), a difficulty which is not entirely obviated by a lateral or oblique position or the employment of selective aortography. However, aortography should be undertaken, and most success will attend the retrograde femoral method with the patient in the oblique position.

There are several aspects of the symptomatology which may produce problems in diagnosis. $\mathrm{Ab}$ dominal pain and weight loss associated with the presence of occult blood in the stool always raises the suspicion of malignancy, and particularly so when the patients being investigated are in middle or late life. In fact, malignancy generally has to be excluded and in the last resort laparotomy may be indicated. If, under such circumstances, routine exploration of the abdomen is negative, the main trunk of the superior mesenteric artery should be exposed and examined.

The post-prandial nature of the pain should not mislead one into diagnosing peptic ulceration. The pain is persistent and unrelieved by alkalis and sedatives. Nevertheless, Mavor and Michie (1958) reported a case which underwent gastric resection. Diarrhoea, when present, and the nature of the stool, point to intestinal malabsorption, and balance studies will confirm this. It is well known that various conditions may cause the malabsorption syndrome, but it is not adequately realized that in later adult life, one of the commoner causes is chronic midgut ischaemia.

\section{Prognosis and Treatment}

From his observations, Mikkelsen (1957) believes that the process in each case is one of unrelenting progression of the disease to the final stage of intestinal gangrene. Certainly the evidence to date suggests that the prognosis is extremely poor and the outcome inevitable. Although there has been one report in the literature in which the patient survived eight years (Berman and Russo, I950), it would seem that the duration of the disease should be measured in weeks or months rather than years. As has been noted, the collateral network is seldom adequate, so that deterioration must always be imminent. This deterioration may occur as a result of local aggravation of the arterial disease or acute occlusion and extension of thrombosis. The latter is the more likely event (Klein, I92 I) and generally follows cardiac decompensation of greater or less degree. Ineffectual laparotomy or abdominal surgery such as gastrectomy may also precipitate acute occlusion in as many as 20 per cent. of all cases of bowel infarction (Johnson and Baggentoss, 1949).

It would appear, then, that prophylactic surgery is on a sound basis. Firstly, because of the severity of pain and the presence of malabsorption the syndrome may in itself demand treatment; and secondly, the prognosis without treatment is poor, bowel infarction being an early complication of high mortality.

The aim of treatment is the restoration of adequate intestinal blood flow, and this can only be achieved by direct surgery on the superior mesenteric artery. Although the technical feasibility of such surgery has been recently emphasize (Mikkelsen, 1957; Mavor and Michie, I9580. Cooke, I958), as yet no successful case has been reported. Mikkelsen (I957) believes that endarterectomy may prove difficult. Shaw and Maynard (1958), however, reported successful restoration of intestinal blood flow in acute mesenteric infarction following thrombo-endarterectomy. In the treatment of stenosis the problem is less acute, and a more elaborate by-pass procedure appears indicated. This may entail using the splenic artery or an arterial homograft, the anastomoses being end to side, and in the latter instance, the by-pass being aorto-mesenteric. Both procedures may prove to be technically easier than transection and reimplantation of the superior mesenteric artery into the aorta (Mikkelsen, 1957).

Much more experience is required before the indications for operation can be clearly defined, and before the operative mortality in both acute and chronic ischaemia assessed. Nevertheless, Shaw and Maynard deserve great credit for demonstrating by successful results, that certain mesenteric vascular lesions can be corrected. Awareness of this possibility may in time show that direct arterial surgery offers the best palliative treatment in this condition and thereby improve the present ominous prognosis and forbidding mortality. 


\section{Summary}

I. Symptoms of chronic midgut ischaemia may result from stenosis or segmental occlusion of the superior mesenteric artery, which is secondary to atherosclerosis. There may or may not be an associated thrombosis of the coeliac axis.

2. Post-prandial abdominal pain, weight loss and altered bowel habit are the characteristic symptoms. In addition there is usually occult blood in the stool and evidence of intestinal malabsorption.

3. The diagnosis is best made on clinical grounds and investigation is likely to prove unrewarding. Aortography has a limited value because of difficulties in interpretation.

4. Direct arterial surgery offers the best method of restoring adequate intestinal blood flow. It is a rational attempt to improve the present poor prognosis and reduce the high mortality that follows treatment by traditional methods.

\section{BIBLIOGRAPHY}

ARKIN, A. (1930), Amer. F. Path., 6, 401 .

BENJAMIN, D. (1948), Amer. F. Surg., 76, 338.

BERMAN, L. G., and RUSSO, F. R. (1950), New Engl. F. Med., 242, 6II.
BURT, C. C., LEARMONTH, J., and RICHARDS, R. L. (1952), Edinb. med. F., 59, 65 .

CARUCCI, J. J. (1953), Amer. F. Surg., 80, 47.

CHIENE, J. (1869), J. Anat. (Paris), 3, 65.

CONNER, L. A. (1933), Amer. F. med. Sci., 185, 31.

COOKE, R. V. (1958), Proc. roy. Soc. Med., 51, 7, 503.

DUNPHY, J. E., and WHITFIELD, R. D. (1940), Amer. F. Surg., 47, 632 .

FORTY, F. (1957), Brit. f. Surg., 44, 458.

HERTZLER, A. E. (1935) ' Surgical Pathology of the Peritoneum.' J. B. Lippincott Co., Philadelphia.

JOHNSON, C. C., and BAGGENTOSS, A. H. (1949), Proc. Mayo Clin., 24, 649.

KLASS, A. A. (1953), f. int. Coll. Surg., 20, 687.

KLEIN, E. (1921), Surg. Gynec. Obstet., 33, 385.

Lancet (1958), June 7, p. 1211.

LARSON, L. M. (1931), Surg. Gynec. Obstet., 53, 54.

LERICHE, R., and MOREL, A. (1948), Ann. Surg., 127, 193.

MCCLENAHAN, J. E., and FISHER, B. (1948), Surgery, 23, 778.

MANDEL, H. N. (1957), New Engl. f. Med., 257, 1035.

MAVOR, G. E., and MICHIE, W. (1958), Brit. med. F., ii, 534.

MIKKELSEN, W. P. (1957), Amer. F. Surg., 94, 262.

MOORE, T. (1941), Brit. F. Surg., 28, 347.

ROB, C. G., and OWEN, K. (1956), Ibid., 44, 247.

SEDLACEK, R. A., and BEAN, W. B. (1957), Ann. intern. Med., 46, 148 .

SEYMOUR, W. B., and LIEBOW, A. A. (1937), Ibid., 10, 1033.

SHAW, R. S., and MAYNARD, E. P. (1958), Nero Engl. F. Med. 258, 874 .

TURNER, W. (1863), Brit. For. med. chir. Rev., 32, 322.

WARBURG (1905), Münch. med. Wschr., 52, 1174.

\section{ANAESTHETICS \\ (Postgraduate Medical Journal)}

Price: 4s. Od. post free

THE MODERN VIEW OF ANAESTHESIA

G. S. W. Organe, M.D., D.A., F.F.A.R.C.S.

THE PRODUCTION OF

UNCONSCIOUSNESS

B. G. B. Lucas, D.A., F.F.A.R.C.S.

ANALGESIA

J. B. Wyman, M.B.E., D.A., F.F.A.R.C.S.

MUSCLE RELAXATION IN SURGERY

Angus Smith, F.F.A.R.C.S.

CONTROL OF THE BLOOD PRESSURE

AND CONTROLLED HYPOTENSION

C. F. Scurr, M.V.O., D.A., F.F.A.R.C.S.

\author{
CHLORPROMAZINE AND ALLIED \\ SUBSTANCES \\ John Beard, M.D., D.A., F.F.A.R.C.S., \\ D.C.H. \\ CONTROLLED HYPOTHERMIA \\ E. J. Delorme, M.D., F.R.C.S.(C.)
MANAGEMENT OF THE APNOEIC PATIENT
Ronald Woolmer, D.A., F.F.A.R.C.S. \\ THE USE OF ANTIDOTES IN \\ ANAESTHESIA \\ B. A. Sellick, D.A., F.F.A.R.C.S.
}

Published by

THE FELLOWSHIP OF POSTGRADUATE MEDICINE

60, Portland Place, London, W.1 